\title{
Applicability of the High Field Model: An Analytical Study Via Asymptotic Parameters Defining Domain Decomposition
}

\author{
CARLO CERCIGNANI $^{\mathrm{a}}$, IRENE M. GAMBA ${ }^{\mathrm{b}}$, JOSEPH W. JEROME ${ }^{\mathrm{c}, *}$ and CHI-WANG SHU \\ ${ }^{a}$ Politecnico di Milano, 20133 Milano, Italy; \\ ${ }^{\mathrm{b}}$ Courant Institute, New York University, New York, NY 10012; \\ ${ }^{\mathrm{c}}$ Department of Mathematics, Northwestern University, Evanston, IL 60208; \\ ${ }^{\mathrm{d}}$ Division of Applied Mathematics, Brown University, Providence, RI 02912
}

\begin{abstract}
In this paper, we present a mesoscopic-macroscopic model of self-consistent charge transport. It is based upon an asymptotic expansion of solutions of the Boltzmann Transport Equation (BTE). We identify three dimensionless parameters from the BTE. These parameters are, respectively, the quotient of reference scales for drift and thermal velocities, the scaled mean free path, and the scaled Debye length. Such parameters induce domain dependent macroscopic approximations. Particular focus is placed upon the so-called high field model, defined by the regime where drift velocity dominates thermal velocity. This model incorporates kinetic transition layers, linking mesoscopic to macroscopic states. Reference scalings are defined by the background doping levels and distinct, experimentally measured mobility expressions, as well as locally determined ranges for the electric fields. The mobilities reflect a coarse substitute for reference scales of scattering mechanisms. See [9] for elaboration.

The high field approximation is a formally derived modification of the augmented drift-diffusion model originally introduced by Thornber some fifteen years ago [25]. We are able to compare our approach with the earlier kinetic approach of Baranger and Wilkins [5] and the macroscopic approach of Kan, Ravaioli and Kerkhoven [20].
\end{abstract}

Keywords: Asymptotic parameters, mesoscopic-macroscopic model, augmented drift-diffusion, high field model, domain decomposition

\section{INTRODUCTION}

This presentation is motivated by the search for more reliable macroscopic models of high field transport in submicron structures which are computationally efficient. This is a research topic of major interest to the micro-electronics industry.

Transport in submicron structures differs from transport in bulk material in many ways. It includes far from equilibrium situations created

\footnotetext{
* Corresponding author
} 
by large electric fields in small structures; ballistic electrons, when dimensions of the drift-forcing term are of the same order as the mean free path; and new effects, introduced by the close proximity to the boundaries of regions where the density gradients are (relatively) rapidly changing.

Most of the previous work on transport in submicron semiconducting structures used several corrections to drift-diffusion equations in which the current is a sum of drift and diffusion terms, where both have field-dependent mobility and diffusion coefficients, respectively. References on models and computational methods for $N^{+}-N-$ $N^{+}$structures of $\mathrm{Si}$ or GaAs can be found in the literature of the last decades [24, 23, 16, 21, 25, 20]. However, the computations have not yielded results which have been genuinely satisfactory, and the main reason has been the lack of understading of the accuracy of the modeling in many heterogeneous structures. An alternative to the drift-diffusion equations has been Monte Carlo simulation, a very costly computational procedure.

An approach intermediate between drift-diffusion and Monte Carlo simulation was generated by the hydrodynamic like theory, using velocity moments of the Boltzmann equation (see $[6,2,3,15]$ ) and realizing closure by imposing constitutive relationships between the state macroscopic variables. Extensive numerical modeling of $N^{+}-N_{-}$ $N^{+} \mathrm{Si}$ and GaAs structures under several geometric constraints can be found in [12, 17, 18, 19]. For the derivation of these models, the distribution function is often assumed to be a Maxwellian distribution. However, this assumption has been shown to be questionable in lightly doped submicron structures, in part because of the presence of ballistic electrons.

However, it has been mathematically proved that hydrodynamic models relax to energy transport models. The latter have been derived as macroscopic limits associated with a particular choice of space-time scale that makes some collision mechanisms dominant (see [10] for a rigorous proof in the case of the relaxation of the full hydrodynamic model). These models, however, cannot describe situations where the drift- forcing term and the resulting scaled mean free path are of the same order, but they are asymptotically correct in regions where the predefined background is very slowly varying (i.e., almost constant doping). A new approach attempting to fill in the middle ground among driftdiffusion, hydrodynamic energy transport, and Monte Carlo simulation (see $[4,5,26]$ ) was the use of direct, numerical solution of the Boltzmann equation within the relaxation time approximation. The use of the Boltzmann equation for non-electron transport is justified in the above references, as well as in [7, 13]. The relaxation time approximation within the Boltzmann-Poisson system approach, positing that all scattering processes can be characterized by a few scattering rates setting up the scale of relaxation time constants, may not be strictly valid for GaAs [11].

Our goal, however, is to present a full solvable model for an $N^{+}-N-N^{+}$structure, as an alternative to all previous calculations, which will produce comparable results with greater efficiency than attained by $[4,5,20]$. In particular, we incorporate into the modeling the macroscopic limit associated with the choice of space, time, potential drop, and local electric field scales, combined with the relaxation time scale, which makes the drift-forcing term and collisions both dominant, and of the same scale set up by the local scaled mean free path.

As a consequence of this approach, we incorporate a level of modeling that takes into account mesoscopic-macroscopic multiscales, and corresponding limit derivations, according to different scales becoming dominant in different regions of the $N^{+}-N-N^{+}$device, depending on the spatial inhomogeneity of the lightly doped region.

\section{PRELIMINARIES OF THE HIGH FIELD MODEL}

We identify three dimensionless parameters, viz.,

- The ratio $\eta$ of drift and free velocity (the latter usually taking on the thermal velocity); 
- The scaled mean free path $\varepsilon$ and

- The scaled Debye length $\gamma$ from the electrostatic potential equation of a self-consistent model.

The dimensionless parameters induce domain dependent macroscopic approximations, which are valid within regimes that do not change the scale of the Debye length. This is of paramount importance in the macroscopic derivation associated with the BTE under a self-consistent field: the scaling of the forcing term depends on the scaled external and internal fields, and must not override the limiting regime of the macroscopic derivation. Therefore, this model incorporates kinetic transition layers linking mesoscopic and macroscopic states.

Reference scalings are defined by the background doping levels and distinct, experimentally measured mobilities which serve to set up the scale of the relaxation time approximation of the scattering mechanisms. In addition, scales take into account locally determined ranges for the electric fields. Numerical experiments of this proposed model are also presented by the authors in [8]. Although this is a first approach to domain decomposition models, based on a mesoscopicmacroscopic linking, our results seem to give an improvement (see also [13]) of computational efficiency, relative to the kinetic computations of Baranger and Wilkins [5]. Moreover, the model itself seems to be an improvement over the augmented drift-diffusion model used by Kan, Ravaioli and Kerkhoven [20]. This model, based on a model introduced empirically by Thornber [25], employs necessary corrections to the usual macroscopic approach to high field models.

\section{BOLTZMANN-POISSON SYSTEM SCALES}

Finally, we concentrate on the derivation of the macroscopic models for semiconductors, based on asymptotic expansions of multiscale dimensionless BTE-Poisson systems. For the sake of simplicity we shall consider only electrons. The semiclassical Boltzmann-Poisson system, within a parabolic band-relaxation time approximation, may be written:

$$
\begin{gathered}
\partial_{t} F+v \cdot \nabla_{x} F-\frac{e}{m} E(x, t) \cdot \nabla_{v} F=\frac{\langle F\rangle M-F}{\tau}, \\
E=-\nabla \phi, \quad \nabla \cdot(\epsilon \nabla \phi)=e\left(\langle F\rangle-N_{d}(x)\right),
\end{gathered}
$$

where $F(x, v, t)$ is the density function for the electron at position $x$, velocity $v$ and time $t$. The constants $e, m, \tau$ and $\epsilon$ represent the electric charge constant, its mass, the relaxation time reference scale, and permittivity of the material, respectively. The functions $\phi$ and $E$ of $(x, t)$ represent the electrostatic potential and its field. The bracket $\langle\cdot\rangle$ denotes the usual average of the distribution function with respect to the velocity variable. Finally $M=M(v, x)$ denotes the Maxwellian centered at zero velocity with $\theta=\theta(x)$ representing the background temperature of the lattice, measured in units of specific energy. Derivations of mesoscopic limits associated with system (1) depend on a particular choice of spacetime scales, collision mechanism scales, and drift velocity scales induced by self-referencing field scales. These regimes, as we shall see, can make some terms of the BTE dominant. The idea that follows then, is to expand the solution of the BTE about the solution of the ground equation corresponding to the dominant terms. Of course, this will depend on the form of scattering mechanisms that set up the scales of the collision terms. Lately, a very nice survey of a hierarchy of macroscopic models for semiconductors under limit regimes that correspond to dominant collision mechanisms has been presented in [1]. These macroscopic models correspond to classical drift-diffusion and so-called energy transport models. They arise from expansion about kernels of the collision operators, which are field independent, so they do not incorporate the scales of the external, or even self-induced electric field. Diffusions are field independent, and consequently they cannot approximate the ballistic electron distribution func- 
tions that have been observed by Barenger and Wilkins [4, 5]. After the work initiated by [14] and [22] on strong forcing scaling for external fields, where the dominant term in the scaled Eq. (1) is given by the balance of the force-drift $(e / m)$ $E(x, t) \nabla_{v} F$ and the collision terms, the following 3scale dimensionless formulation has been formulated in [9] in $\ell$ Euclidean dimensions:

$$
\begin{gathered}
\eta F_{t}+v \cdot \nabla_{x} F+\frac{\eta}{\varepsilon} \nabla_{x} \phi \cdot \nabla_{v} F=\frac{1}{\varepsilon}\{\langle F\rangle M-F\}, \\
\Delta \phi=\gamma\left(\frac{1}{\eta^{\ell}}\langle F\rangle-N_{d}(x)\right),
\end{gathered}
$$

where $\varepsilon=\frac{\tau \bar{\theta}^{1 / 2}}{L}$ is the scaled mean free path for a length scale $L$ and relaxation time $\tau$, and $\bar{\theta}$ is the reference scale of the background energy. The dimensionless constant $\eta=U / \bar{\theta}^{1 / 2}$, where $U$, in units of velocity, is given by $\frac{\tau e}{m} \frac{[\phi]}{L}$, takes into account the reference scale of the local electric field. Finally, the dimensionless constant $\gamma=\frac{\bar{\rho} e L^{2}}{\epsilon \mid \phi]}$ represents the scaled Debye length, where $\bar{\rho}$ scales the density of the fixed background $N_{d}(x)$.

Now, a strong force regime corresponds to values of $\eta=O(1), \gamma=O(1)$ and $\varepsilon<L$. In this case, system (2) is obtained, where the distribution function $F$ is scaled with the drift velocity $U$ (instead of the low field scalings that use the thermal velocity scale for $F$ ). The time scale is then fixed at $L U^{-1}$.

\section{ASYMPTOTIC LIMITS AND THE HIGH FIELD MODEL}

Clearly, any asymptotic expansion is justified under the assumption that the scaling does not break down. Thus, any expansion of the BTEPoisson system (2) requires that the scale of the internal field defined by the Poisson equation (i.e., depending on the value of $\gamma$ ) does not override the scaling of the BTE.

$\bar{\rho}$ is the reference scale of the predefined $N_{d}(x)$ and will change scale whenever $N_{d}(x)$ does. Accordingly, so will the local field. Therefore, as an immediate consequence of the fact that $\gamma=\gamma(\bar{\rho})$ can be easily checked for GaAs (as in $[5,20])$, the dimensionless quantities vary in the $\mathrm{N}^{+}$regions from the $N$ regions. Thus, in $\mathrm{N}^{+}$regions, $\eta=\varepsilon=o(1)$. Because of scaling times and the distribution function $F$, accordingly, (2) yields the Drift-Diffusion-Energy Transport regime as the relative electric field remains moderate and the collision mechanisms become dominant.

However, in the $N$ region it is computed by the authors in [8] that $\varepsilon \approx(1 / 7)$ and $\eta=O(1)$. Thus, while $\gamma$ remains of order $O(1)$, it has been shown that densities and the electric field satisfy the equations,

$$
\begin{gathered}
\rho_{t}+\nabla \cdot J=0, \\
J=-\tau\left[\nabla_{x}\left(\rho\left(\theta+\mu^{2} E \otimes E\right)\right)\right]-\mu \rho E \\
+\tau \mu E \rho \nabla \cdot(\mu E)-\tau \mu \rho \frac{e}{\epsilon}(\mu \rho E-w), \\
\nabla \cdot w=0, \quad \operatorname{curl} w=\mu E \otimes \nabla \rho, \\
E=-\nabla \phi, \quad \nabla \cdot(\epsilon \nabla \phi)=\left(\rho-N_{d}(x)\right),
\end{gathered}
$$

in their dimensionalized formulation. Here $\mu$ is the mobility. The new variable $w$ can be interpreted as the curl of a magnetic field, associated with the high electric current. This model is a correction of the one proposed by Thornber [25], where the diffusion and transport coefficients were given empirically.

We point out that in this relaxation time approximation, the mobility coefficient is given by $\mu=\frac{e \tau}{m}$, so clearly the saturation of the mobility depends on how the scattering rates scale for $\tau$ changing with respect to the electric field. The standard assumption based on curve fitting is to take $\mu=C_{1} /\left(1+C_{2}|E|^{2}\right)^{1 / 2}$ (see [16]).

We stress that this regime is valid only for the strong force scaling. The transition regime from weak to strong forcing scaling, and conversely, ought to be done by carefully considering the corresponding kinetic transition layers. The implementation and solution of this transition problem require solution of boundary value problems at the kinetic and macroscopic level as well. 


\section{DOMAIN DECOMPOSITION AND TRANSITION LAYERS}

Appropriate boundary data at the kinetic level and the corresponding limiting data for the mesoscopic approximation are presented in [9]. For kinetic level distribution functions there are prescribed exact neutral space charge conditions at contact boundaries and standard insulating boundary conditions at insulating walls. Then, these conditions, together with the asymptotic approximations, yield Dirichlet boundary conditions at the macroscopic level, with a correction term of the order of the asymptotic parameter (i.e., the order of the scaled mean free path $\varepsilon$ ). The contact condition, at the kinetic level, on a space boundary section $\Gamma$ reads [9]:

$$
F_{-}=\left(N_{d}(x)-\int_{v \cdot n>0} F_{+} d v\right) \frac{P}{\begin{array}{c}
v \cdot n<0 \\
\text { on } v \cdot n<0,
\end{array}}
$$

where $n$ denotes the outer normal with respect to the boundary section $\Gamma$, and $P$ denotes the lowest order term in the expansion for either weak force scaling (i.e., $P$ is a Maxwellian) or strong force scaling (i.e., $P$ peaks asymmetrically and is electric field dependent).

The corresponding macroscopic condition for the inner boundary can be computed explicitly [9] and yields

$$
\begin{array}{r}
\rho(x)=N_{d}(x)+o(\varepsilon) K(x), \\
K(x)=\frac{\left\langle v F_{-}^{(1)}\right\rangle \cdot n\left\langle F_{-}\right\rangle}{\left\langle v \cdot F_{-}^{(0)}\right\rangle \cdot n} .
\end{array}
$$

Here,

$$
\begin{array}{r}
F \simeq F^{(0)}+\varepsilon F^{(1)}+O\left(\varepsilon^{2}\right), \quad F^{(0)}=\langle F\rangle P, \\
\left\langle f_{-}\right\rangle=\int_{v \cdot n<0} f d v .
\end{array}
$$

We finally remark that the Ohmic contacts on $\mathrm{N}^{+}$regions correspond to a local scaled mean free path $\varepsilon$ of much smaller order than in the $N$ regions. Hence, at boundary ohmic contacts on $\mathrm{N}^{+}$regions, condition (5) is almost a neutral space charge condition at the macroscopic level, as historically has been, and still is typically prescribed. However, at the transition layer from the $\mathrm{N}^{+}$to the $N$ regime, the transfer of the data entails a kinetic computation using condition (4); then, one prescribes an exact background density, and thus delivers, at the next level, for the strong force model, a condition for the density that is an $O(\varepsilon)$ derivation from the background density. This fact can actually be observed in the computations of Barenger and Wilkins [4, 5].

Numerical implementations of strong-weak forcing decomposition are presented by the authors [8] in this same issue. However, we use there a matching with hydrodynamic computations of well accepted performance. In Figure 3 of [8], we present a graph of $\eta$, which clearly correlates with the suggested domain decomposition. Theoretical justifications and alternative numerical methods of these domain decomposition ideas are underway and will be presented in future work.

\section{Acknowledgements}

The second author is supported by the National Science Foundation under grant DMS-9623037. The third author is supported by the National Science Foundation under grant DMS-9424464. The fourth author is supported by the National Science Foundation under grants ECS-9214488 and ECS-9627849, and the Army Research Office under grant DAAH04-94-G-0205.

\section{References}

[1] Ben Abdallah, N. and Degond, P. (1996). On a hierarchy of macroscopic models for semiconductors. J. Math. Phys., 37, 3306-3333.

[2] Anile, A. M. and Muscato, O. (1995). Improved hydrodynamical model for carrier transport in semiconductors. Phys. Rev. B, 51, 16728-16740.

[3] Baccarani, G. and Wordeman, M. R. (1985). An investigation of steady-state velocity overshoot effects in Si and GaAs devices. Solid State Electr., 28, 407-416. 
[4] Barenger, H. U. and Wilkins, J. W. (1984). Ballistic electrons in an inhomogeneous submicron structure: Thermal and contact effects. Phys. Rev. B, 30, 7349-7351.

[5] Barenger, H. U. and Wilkins, J. W. (1987). Ballistic structure in the electron distribution function of small semiconducting structures: General features and specific trends. Phys. Rev. B, 36, 1487-1502.

[6] Blotekjaer, K. (1970). Transport equations for electrons in two-valley semiconductors. IEEE Trans. Electron Devices, 17, $38-47$.

[7] Cercignani, C. (1987). The Boltzmann Equation and its Application. Springer-Verlag, New York.

[8] Cercignani, C., Gamba, I. M., Jerome, J. W. and Shu, C.-W., Applicability of the high field model: A preliminary numerical study. This volume.

[9] Cercignani, C., Gamba, I. M. and Levermore, C. D., (1997). High field approximations to Boltzmann-Poisson system boundary conditions in a semiconductor. Appl. Math. Lett., 10, $111-117$.

[10] Chen, G.-Q., Jerome, J. and Zhang, B., Existence and the singular relaxation limit for the inviscid hydrodynamic energy model. Proceedings, Workshop on Modeling and Computation for Applications in Mathematics, Science and Engineering, Oxford University Press, to appear.

[11] Conwell, E. (1982). Handbook of Semiconductors, 1 (W. Paul, ed.), p. 513, North-Holland.

[12] Fatemi, E., Jerome, J. and Osher, S. (1991). Solution of the hydrodynamic device model using high-order nonoscillatory shock capturing algorithms. IEEE Trans. Computer-Aided Design of Integrated Circuits and Systems, 10, 232-244.

[13] Ferry, D. K. and Grondin, R. O. (1991). Physics of Submicron Devices. Plenum Press, New York.

[14] Frosali, G., van der Mee, C. V. M. and Paveri Fontana, S. L. (1989). Conditions for run-away phenomena in the kinetic theory of particle swarms. J. Math. Phys., 3, 11771186.

[15] Gnudi, A., Odeh, F. and Rudan, M. (1988). An efficient discretization scheme for the energy continuity equation in semiconductors. In Proceedings of SISDP, 387-390.

[16] Jerome, J. W. (1996). Analysis of Charge Transport; A Mathematical Theory of Semiconductor Devices. Springer.

[17] Jerome, J. W. and Shu, C.-W. (1994). Energy models for one-carrier transport in semiconductor devices. In Semiconductors, Part II, 59 of IMA Volumes in Mathematics and its Applications (W. M. Coughran, J. Cole, P. Lloyd and J. K. White, eds.), pp. 185-207. Springer-Verlag, New York.

[18] Jerome, J. W. and Shu, C.-W. (1995). The response of the hydrodynamic model to heat conduction, mobility, and relaxation expressions. VLSI DESIGN, 3, 131-143.

[19] Jerome, J. W. and Shu, C.-W. (1995). Transport effects and characteristic modes in the modeling and simulation of submicron devices. IEEE Trans. Computer-Aided Design of Integrated Circuits and Systems, 14, 917-923.

[20] Kan, E. C., Ravaioli, U. and Kerkhoven, T. (1991). Calculation of velocity overshoot in submicron devices using an augmented drift-diffusion model. Solid-State Electr., 34, 995-999.

[21] Markowich, P. A., Ringhofer, C. A. and Schmeiser, C. (1990). Semiconductor Equations. Springer-Verlag, Vienna.

[22] Poupaud, F. (1992). Runaway phenomena and fluid approximation under high fields in semiconductor kinetic theory. Z. Angew. Math. Mech., 72, 359-372.
[23] Selberherr, S. (1984). Analysis and Simulation of Semiconductor Devices. Springer-Verlag, New York.

[24] Sze, S. M. (1981). Physics of Semiconductor Devices. John Wiley, New York.

[25] Thornber, K. K. (1983). Current equations for velocity overshoot. IEEE Electron Device Lett., 3, 69-71.

[26] Trugman, S. A. and Taylor, A. J. (1986). Analytic solution of the Boltzmann equation with applications to electron transport in inhomogeneous semiconductors. Phys. Rev. $B, 33,5575-5584$.

\section{Authors' Bigraphies}

Carlo Cercignani received his Univ. Milano Laurea Physics in 1961 and his Univ. Milano Laurea Mathematics in 1963. From 1963 to 1966 he was Assistant and Associate Professor of Plasma Physics at the University of Milano. He was a Visiting Associate Professor of Applied Mathematics at M.I.T. during 1966-67. He came to Politecnico di Milano in 1968 and has been Professor in Theoretical Mechanics since 1975. His scientific specialties include the kinetic theory of gases and its applications to rarefied gas dynamics, neutron transport and semiconductors; nonequilibrium statistical mechanics, fluid dynamics and singular integral equations. His current research interests are analytical and numerical treatment of kinetic and transport equations for gases and semiconductors. $\mathrm{He}$ is a member of Accademia Nazionale dei Lincei, Istituto Lombardo and Académie des Sciences de Paris. In addition he is a member of the National Committees for Mathematics of the Italian Research Council (C.N.R., President), Scientific Committee of the National Group for Mathematical Physics (G.N.F.M.) of C.N.R., a member of General Assembly of IUTAM, General Assembly of IMU, Congress Committee of IUTAM, Scientific Committee of 1st European Mathematical Congress, and Advisory Committee for Symposia on Rarefied Gas Dynamics. He serves on the following editorial boards (1993): Fluid Dynamics Research, Transport Theory and Statistical Physics, MECHANICS Research Communications, Annali di Matematica Pura e Applicata, Applied Mathematics Letters, European Journal of Me- 
chanics B (Ass. Ed.), Nonlinear Differential Equations and Applications, Surveys in Mathematics for Industry, Continuum Mechanics and Thermodynamics. He has authored or coauthored seven scientific books and more than two hundred scientific publications. $\mathrm{He}$ is the recipient of the Gold Medal for Mathematics of the Accademia dei XL (1982), Prize “Citta' di Cagliari" for Applied Mathematics (1992), Docteur Honoris Causa of the University Pierre et Marie Curie (Paris VI) (1992) and the Humboldt Prize (Academic Year 1995-1996).

Irene M. Gamba received the Ph.D. degree in Mathematics from The University of Chicago in 1989. From 1990 to 1991 she was at Purdue University as a Visiting Assistant Professor and Research Associate. She was an Assistant Professor at The College of New Jersey, Trenton, NJ, during 1991-92. She came to the Courant Institute of Mathematical Sciences, New York University, in 1992. There she was an NSF Post doctoral Fellow during 1992-94, an Assistant Professor from 1994-96 and Associate Professor during 1996-97. She has accepted a position as Professor of Mathematics at the University of Texas at Austin beginning in August, 1997. Her research interests include nonlinear analysis applied to gas dynamics and charged-particle transport systems in the mathematical modeling of microelectronic devices.

Joseph W. Jerome received the Ph.D. degree in Mathematics from Purdue University in 1966. He was visiting Assistant Professor at the Mathematics Research Center, University of Wisconsin, during 1966-68 and was Assistant Professor at
Case Western Reserve University during 196870. He joined Northwestern University in 1970, where he has been Professor of Mathematics and Applied Mathematics since 1976 . He has held sabbatical positions at Oxford University, England, 1974-75, University of Texas, 1978-79 and Bell Laboratories, Murray Hill, 1982-83. He was visiting scholar at the University of Chicago in 1985. He received the Distinguished Alumnus Award from Purdue University's School of Science in 1996. His research interests include applied analysis, numerical analysis, computational electronics, and ion transport in biology. The most recent of his three books, Analysis of Charge Transport, was published by Springer in 1996.

Chi-Wang Shu received the B.S. degree in Mathematics from the University of Science and Technology of China (USTC) in 1982 and the Ph. D. degree in Applied Mathematics from the University of California at Los Angeles (UCLA) in 1986. He was a post doctoral fellow at the Institute for Mathematics and Its Applications (IMA), University of Minnesota, during 19861987 and came to Brown University in 1987, where he has been Professor of Applied Mathematics since 1996. He was a co-recipient of the Chinese Academy of Science Award in Numerical Analysis and Scientific Computing in 1995. His research interests include numerical analysis, scientific computing and computational physics. He currently serves on the editorial boards of Mathematics of Computation, SIAM Journal on Numerical Analysis and Journal of Computational Mathematics. 

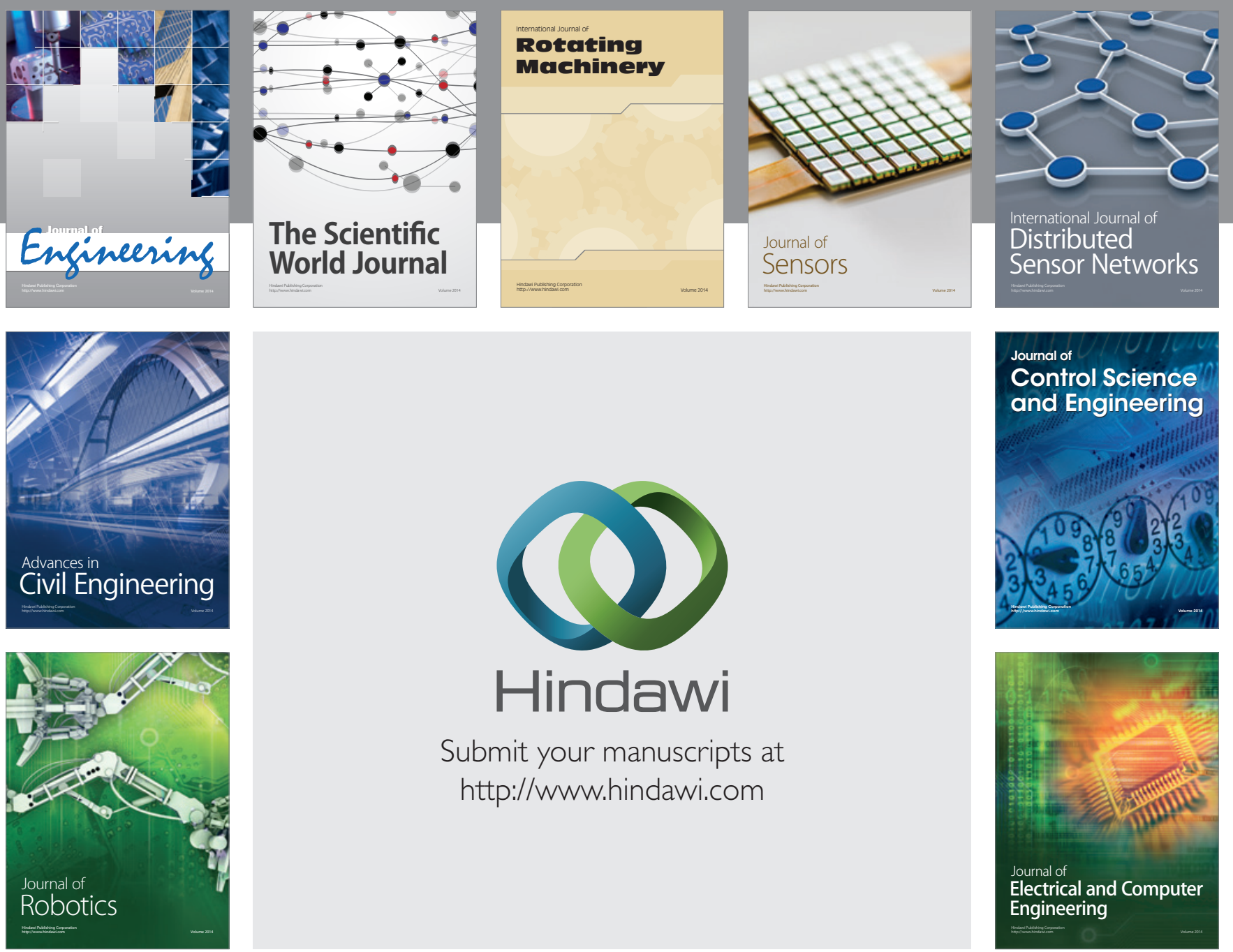

Submit your manuscripts at

http://www.hindawi.com
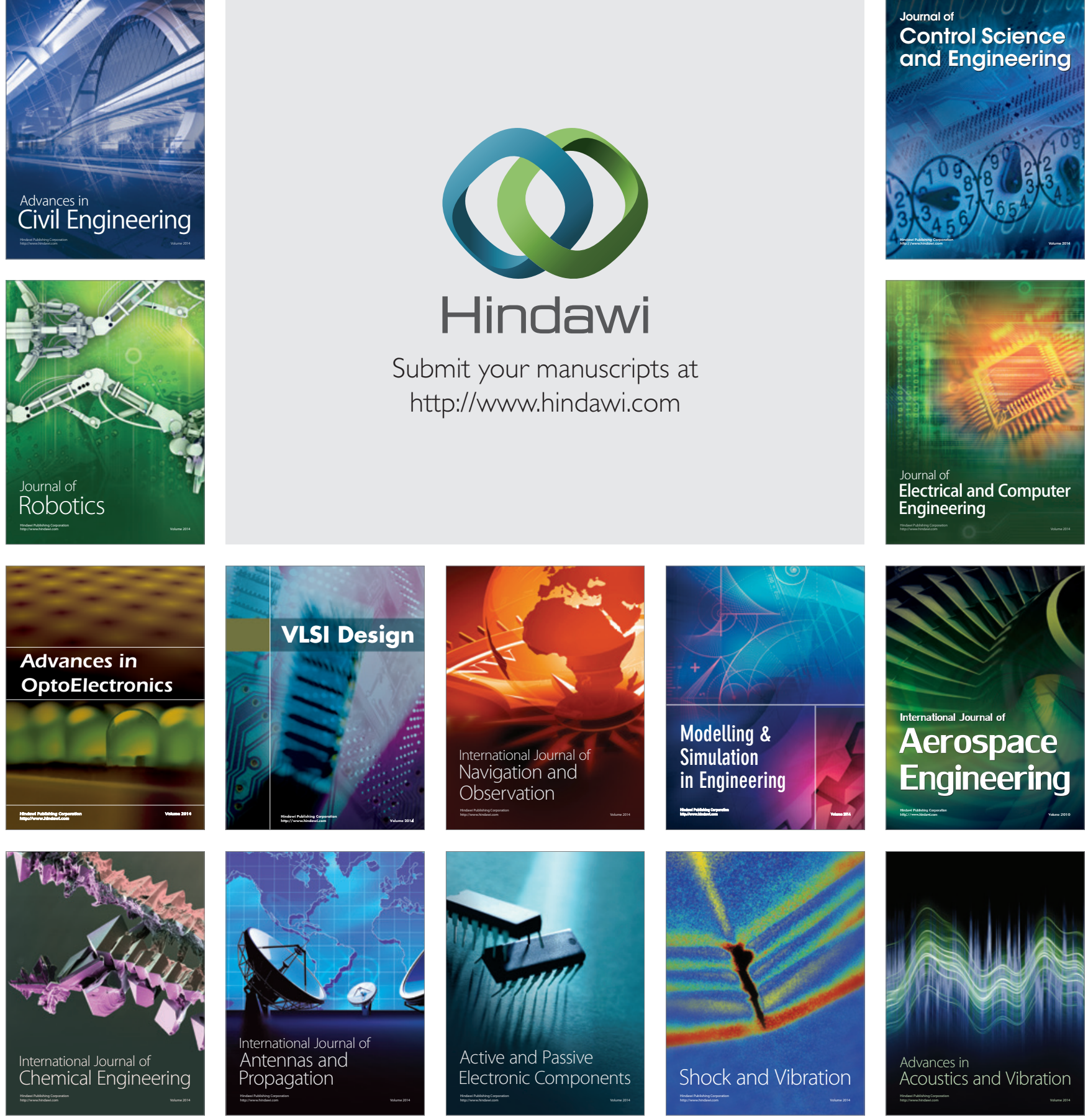